\title{
Congratulations to Kurt Wüthrich for his Nobel Prize in Chemistry
}

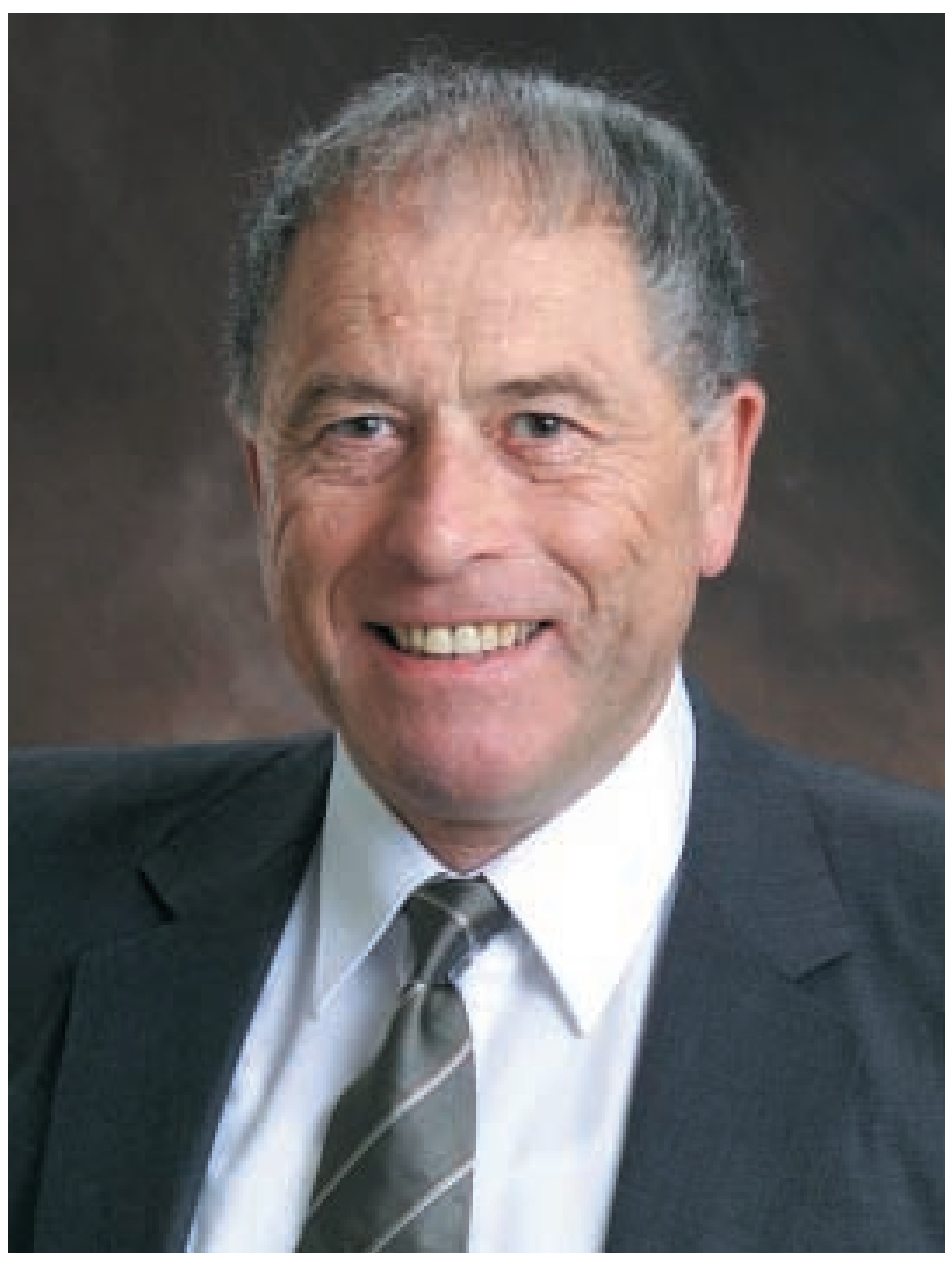

Since many years, insiders unanimously agreed Kurt Wüthrich deserves the great prize! His and his group's innovations have changed the field of molecular biology, and the influence on the life sciences in general is immense. Before, it was impossible to study the inner workings and the interactions of biomolecules in their natural media, in aqueous solution or within membranes. Today, we have access to more secrets of nature than ever before, and our understanding of life processes and our ability to cure diseases have grown immensely.

It seems to be common to all great innovations and achievements: The underlying idea is very simple, almost trivial; and over and over again colleagues ask themselves, why didn't I have that seminal idea before? - Indeed, the seminal idea of Kurt Wüthrich is very simple, and intriguing at the same time: Take two recently introduced new techniques, give them wellsounding, attractive names, combine them in an innovative, ingenious manner so that one plus one is infinitely more than two, and, finally, apply the novel methodology to a large number of highly relevant problems of great actuality. This is, in a nut shell, Kurt Wüthrich's secret of success. But it does not work in everybody's hands; one needs in addition his perseverance, his enthusiasm and drive, and his unbreakable belief in the ultimate success of his approach.

The two above-mentioned ingredients for determining biomolecular structures were two novel two-dimensional NMR techniques, COSY and NOESY, employing Kurt Wüthrich's catchy terminology; COSY for tracing out internuclear connectivity through chemical bonds and determining dihedral bond angles, and NOESY for measuring internuclear distances through space. And the combination of the two experiments led to his ingenious procedure for sequential assignment of the nuclear magnetic resonances of backbone protons in proteins, and later also in nucleic acids. The results became the inputs of sophisticated computer routines that, finally, calculate the best fitting three-dimensional molecular structures.

This procedure is today a standard tool, indispensable in any advanced molecular biology laboratory. Kurt Wüthrich's research group solved during the past twenty 
years by NMR structural questions for many proteins that could not adequately be crystallized, a prerequisite for applying X-ray crystallography.

Both experiments, COSY and NOESY, were originally invented by Jean Jeener, a brilliant Belgium physicist, without yet conceiving their final names. In 1971, Jean Jeener proposed the very first twodimensional correlation experiment during a lecture at an AMPERE Summer School at Basko Polje in Yugoslavia. This experiment for tracing out nuclear spin systems was subsequently implemented in the author's laboratory in 1974 and introduced 1977 into Kurt Wüthrich's research group in the hope of possible biological applications. The second experiment was suggested in 1977 again by Jean Jeener during a private discussion at a Gordon conference in Wolfeboro at the beautiful lake Winnipesaukee, New Hampshire. Its purpose was to trace out networks of nuclear relaxation processes. Again, the author's research group attempted its implementation, at first for elucidating chemical exchange networks, which turned out to be easier than exploring relaxation. But soon afterwards, 1980, Anil Kumar discovered in a collaborative research project, carried out in Wüthrich's research group, that the experiment works beautifully also for cross relaxation if sufficiently large molecules are used as targets.

At this moment, Kurt Wüthrich became fully alert to the possible relevance of the two independent experiments. Their marriage was soon sealed for all time to come, and their naming ceremony happened at the same time, actually to the author's dismay, being afraid of insulting the purist minds of scientists. But he soon converted also to Wüthrich's trendy nomenclature.

Although the author's collaboration with Kurt Wüthrich continued for several more years, the seminal further development happened almost entirely within Wüthrich's group. As usual, the dry spell was long from the first conception of the idea to its truly operative implementation, and it took enormous efforts, both on the experimental spectroscopic side as well as for conceiving powerful computer data processing routines. And the first protein structure, the one of proteinase inhibitor IIa from bull seminal plasma, obtained entirely by two-dimensional NMR, could be published in 1985.

At this early stage in two-dimensional biological NMR, there was hardly any serious competition world-wide, and never any doubt occurred that the priority for the sequential assignment of proteins and their structure determination by NMR belongs to
Wüthrich's research group. But rather rapidly, other research teams acquired the necessary knowledge, and serious competition arouse. Nevertheless Wüthrich's group has always kept the lead until today.

A large number of exceedingly beautiful and highly relevant biomolecular structures have been determined in the mean time by his research group. Just three outstanding examples shall be mentioned: the three-dimensional structure of the complex of the peptide cyclosporin and the protein cyclophilin, of importance for the suppression of the immune response during organ transplantations; the geometry of the DNA complex of the Antennapedia homeodomain, a transcription factor from the organism Drosophila menogaster; and the structures of the mouse, bovine, and human prion proteins, of major significance for understanding the inherent processes involved in BSE and CJD diseases.

Why has NMR been so successful in Zürich? First of all, certainly because Kurt Wüthrich decided 1969 to come to the ETH, invited by the Professors Hans $\mathrm{H}$. Günthard and Robert Schwyzer, after his successful postdoctoral years in the United States where he became, for the first time, fascinated by the potential of NMR as applied to biomolecules. - But in addition, NMR had already long before gained a very strong footing in Zürich. The first research in nuclear magnetic resonance was performed at the physics department of the University of Zürich. Hans Staub spent his postdoctoral years at Stanford in the group of the Swiss citizen, Nobel Laureate, and ETH alumnus Felix Bloch. After being appointed 1949 as professor of physics, he established a very lively and successful group in NMR. Later, 1961, Professor W.H. Heini Gränicher employed NMR at the physics department of ETH for seminal studies on ice. The first pioneering introduction of NMR into chemistry at Zürich happened 1957 by Professor Hans H. Günthard and especially by Professor Hans Primas who designed innovative high-resolution NMR instrumentation. This formed the major germ for the future Nobel successes at ETH Zürich, and also for the commercialization of NMR through the company Trüb-Täuber, which led to the later foundation of the internationally leading company Spectrospin, known today under the name Bruker BioSpin.

Often, serendipity is made responsible for scientific success, but we know at least as many examples where from the beginning a clearly defined goal was pursued without compromise. Wüthrich's success undoubtedly belongs to this second category. He knew his goal, the determination of biomolecular structures, already very early and was systematically looking for suitable tools, enabling him to attain the set goal. Obviously, each scientist also requires luck in his career. And Wüthrich's luck was that the proper tools just became available when he needed them. But it is to his merit that he realized their suitability and improved them to their perfection.

Wüthrich's achievements are an excellent example for 'oriented basic research', research that is of a fundamental nature and nevertheless has clear set practical goals. Wüthrich was never caught within a detached ivory tower. His type of research can most easily be justified towards the funding agencies and towards the general public. It is also the kind of research that immediately fascinates young adventurous scientists because it is interesting, challenging, and useful, at the same time. It is not astonishing that numerous renowned scientists started their successful careers within Wüthrich's group.

Let us hope that Wüthrich's example is contagious for the entire scientific community, with the effect that university research continues to contribute significantly to solving problems of societal importance.

We would like to congratulate our colleague Kurt Wüthrich to his Nobel Prize in Chemistry.

\section{Richard R. Ernst}

Laboratorium für Physikalische Chemie ETH Hönggerberg HCI

CH-8093 Zürich 